\title{
BMJ Open Artificial intelligence mobile health platform for early detection of COVID-19 in quarantine subjects using a wearable biosensor: protocol for a randomised controlled trial
}

Chun Ka Wong (D) , ${ }^{1}$ Deborah Tip Yin Ho, ${ }^{2}$ Anthony Raymond Tam, ${ }^{2}$ Mi Zhou (D) , Yuk Ming LAU, ${ }^{1}$ Milky Oi Yan Tang, ${ }^{2}$ Raymond Cheuk Fung Tong, ${ }^{3}$ Kuldeep Singh Rajput, ${ }^{4}$ Gengbo Chen, ${ }^{5}$ Soon Chee Chan, ${ }^{5}$ Chung Wah SIU, ${ }^{1}$ Ivan Fan Ngai Hung ${ }^{2}$

To cite: Wong CK, Ho DTY Tam AR, et al. Artificial intelligence mobile health platform for early detection of COVID-19 in quarantine subjects using a wearable biosensor: protocol for a randomised controlled trial. BMJ Open 2020;10:e038555. doi:10.1136/ bmjopen-2020-038555

- Prepublication history and additional material for this paper are available online. To view these files, please visit the journal online (http://dx.doi org/10.1136/bmjopen-2020038555).

Received 17 March 2020 Revised 24 June 2020 Accepted 26 June 2020

Check for updates

(C) Author(s) (or their employer(s)) 2020. Re-use permitted under CC BY-NC. No commercial re-use. See rights and permissions. Published by BMJ.

For numbered affiliations see end of article.

Correspondence to

Dr Chung Wah SIU;

cwdsiu@hku.hk

\section{ABSTRACT}

Introduction There is an outbreak of COVID-19 worldwide. As there is no effective therapy or vaccine yet, rigorous implementation of traditional public health measures such as isolation and quarantine remains the most effective tool to control the outbreak. When an asymptomatic individual with COVID-19 exposure is being quarantined, it is necessary to perform temperature and symptom surveillance. As such surveillance is intermittent in nature and highly dependent on self-discipline, it has limited effectiveness. Advances in biosensor technologies made it possible to continuously monitor physiological parameters using wearable biosensors with a variety of form factors.

Objective To explore the potential of using wearable biosensors to continuously monitor multidimensional physiological parameters for early detection of COVID-19 clinical progression.

Method This randomised controlled open-labelled trial will involve 200-1000 asymptomatic subjects with close COVID-19 contact under mandatory quarantine at designated facilities in Hong Kong. Subjects will be randomised to receive a remote monitoring strategy (intervention group) or standard strategy (control group) in a 1:1 ratio during the 14 day-quarantine period. In addition to fever and symptom surveillance in the control group, subjects in the intervention group will wear wearable biosensors on their arms to continuously monitor skin temperature, respiratory rate, blood pressure, pulse rate, blood oxygen saturation and daily activities. These physiological parameters will be transferred in real time to a smartphone application called Biovitals Sentinel. These data will then be processed using a cloud-based multivariate physiology analytics engine called Biovitals to detect subtle physiological changes. The results will be displayed on a web-based dashboard for clinicians' review. The primary outcome is the time to diagnosis of COVID-19.

Ethics and dissemination Ethical approval has been obtained from institutional review boards at the study sites. Results will be published in peer-reviewed journals.
Strengths and limitations of this study

- Automatic physiological data collection by wearable biosensors that do not rely on self-discipline of quarantined subjects.

- Large number of physiological parameters monitored, including skin temperature, respiratory rate, blood pressure, pulse rate, blood oxygen saturation and daily activities, instead of relying solely on temperature measurement.

- Continuous data collection and analysis throughout the day.

- Limited by being single-centre based and exploratory in nature.

\section{INTRODUCTION}

COVID-19 emerged in December 2019, ${ }^{1-4}$ and in a mere few months has spread to more than 104 countries, resulting in an outbreak of viral pneumonia worldwide. As of 12 March 2020, the virus has reportedly caused 127863 infections and 4718 deaths globally. ${ }^{5}$ The WHO declared COVID-19 a public health emergency of international concern on 30 January 2020 and further characterised it as a pandemic on 11 March 2020. As there is no effective therapeutic or vaccine for the condition yet, rigorous implementation of traditional public health measures such as isolation, quarantine, social distancing and community containment is the principle strategy used to control the COVID-19 epidemic. ${ }^{6}$ In addition to isolating patients with confirmed COVID-19 infection from the non-infected population, it is equally if not more important to quarantine asymptomatic individuals with COVID-19 exposure in order to reduce viral spread in the community. 


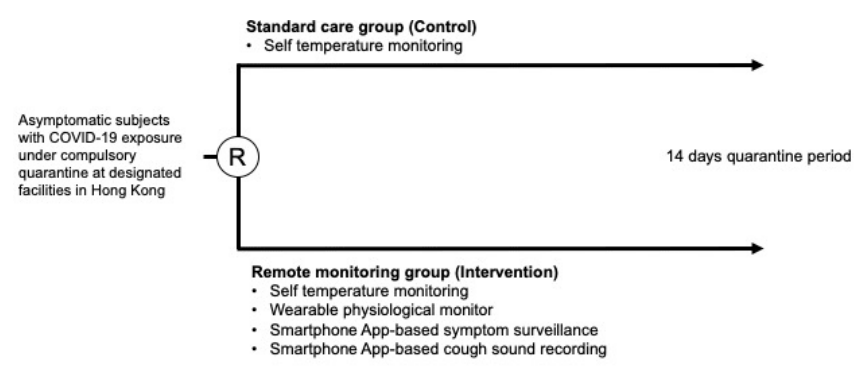

Primary endpoint: the time to detection of COVID-19 infection using nasopharyngeal sampling for COVID-19 RT-PCR Figure 1 Study flow.

Indeed, quarantine measures have been initiated in many countries and regions to restrict movement of asymptomatic individuals with COVID-19 exposure in the community. These individuals are required to stay at home or at designated quarantine facilities. In addition, they are also asked to perform fever and symptom surveillance during the presumed incubation period (14 days). However, as such surveillance is intermittent in nature and highly dependent on self-discipline, it has limited effectiveness Furthermore, it has been reported that as many as $50 \%$ of COVID-19 infected patients had no fever until developing full-blown disease, ${ }^{3} 7$ thereby body temperature surveillance per se may not be sufficient to detect early disease progression.

In the past few decades, advances in technology has allowed progressive miniaturisation of electronic biosensors such that they can be incorporated into wearable devices to allow continuous monitoring of physiological parameters such as skin temperature, heart rate, respiratory rate, oxygen saturation, perspiration and activity of ambulatory subjects in a $24 / 7$ basis. ${ }^{89}$ Together with state-of-the-art telecommunication technologies that allow instantaneous and multidirectional massive data transfer, it is now possible to remotely monitor physiological parameters of a large number of subjects in real time. These data can subsequently be relayed to physicians to allow timely intervention. Nonetheless, the potentials of using wearable biosensors to improve disease management have not been fully explored in real world settings. The current study aims to assess the impact of performing continuous remote monitoring of asymptomatic subjects with COVID-19 exposure under mandatory quarantine at designated facilities in Hong Kong using the Biovitals Sentinel platform. The platform consists of a clinicalgrade wearable biosensor worn on the upper arm called Everion (Biofourmis, Singapore) which allows continuous multidimensional physiological parameters monitoring and an artificial intelligence-powered physiology analytical platform Biovitals (Biofourmis, Singapore) for detecting disease progression. The research hypothesis is that by collecting continuous physiological data using Biovitals Sentinel and patient-reported outcomes, and processing them with a cloud-based analytics platform, Biovitals, it will be possible to identify physiology changes
Box 1 Inclusion and exclusion criteria

Inclusion criteria

- Age $\geq 18$ years.

- Asymptomatic for COVID-19 pneumonia.

- With close COVID-19 contact under mandatory quarantine at designated facilities in Hong Kong.

- Voluntarily agrees to participate by providing written informed consent.

\section{Exclusion criteria}

- Symptoms suggestive of COVID-19 infection including fever, upper respiratory symptoms and/or gastrointestinal symptoms at recruitment.

- Confirmed COVID-19 infection.

- Planned laboratory test for COVID-19.

- Inability or refusal to provide informed consent.

Lack of skills in operating simple electronic devices.

and detect other clinically meaningful alerts that will indicate early clinical progression in quarantined subjects with COVID-19 exposure.

\section{METHODS AND ANALYSIS}

This clinical trial protocol follows Standard Protocol Items: Recommendations for Interventional Trials. ${ }^{10} 11$ The underlying protocol follows Consolidated Standards of Reporting Trials. ${ }^{12}{ }^{13}$ The clinical trial was registered on clinicaltrials.gov (NCT04343794).

\section{Study design and participants}

This is a prospective, randomised-controlled, openlabelled study. Asymptomatic subjects with COVID-19 exposure at designated facilities under the compulsory quarantine measure introduced on 8 February 2020 in Hong Kong, fulfilling inclusion and exclusion criteria of the study will be invited to participate in this study. Box 1 summarises the inclusion and exclusion criteria.

There will be two phases of subject recruitment. The initial run-in phase is a single-arm, open-label study design confirmation phase with approximately 100 subjects. All subjects will undergo remote physiological monitoring using the Biovitals Sentinel platform. The data collected from the platform and clinical data from the health system will be used to validate the solution and the study design for the randomised phase. In the subsequent randomised phase, subjects will be randomised in a $1: 1$ ratio to $^{1}$ continuous remote physiological monitoring using the Biovitals Sentinel platform (intervention group) or $^{2}$ usual care (control group) during the 14-day quarantine period (figure 1).

In addition to daily body temperature monitoring and symptom surveillance in the control group, subjects randomised to the intervention group will wear a multisensor-based wearable armband biosensor, Everion, during the quarantine period. Everion is a wearable vital sign monitor capable of tracking heart rate, heart rate variability, blood oxygen saturation, blood pulse wave, 


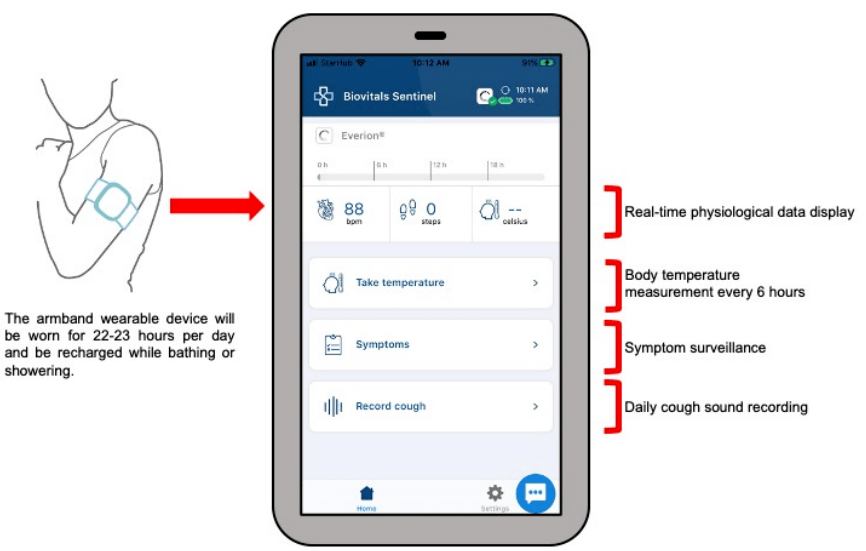

Figure 2 Wearable device and home screen of the dedicated smartphone application.

respiration rate at rest, skin blood perfusion, activity, steps, skin temperature, barometric pressure and electrodermal activity. The wearable biosensors will be worn for 22-23 hours per day and be recharged while bathing or showering. In addition, patients will be instructed to report their symptoms and record their cough sounds using the specially designed smartphone application daily. The physiological parameters obtained will be automatically transferred in real time through a specially designed smartphone application to a secured cloud storage for processing using the Biovitals Analytics Engine. The results will be displayed on a web-based clinician's dashboard for review (figures 2-5). The Biovitals Analytics Engine will process these multidimensional physiology parameters to detect subtle physiological changes preceding critical events, thereby enabling clinicians to promptly review and intervene. As an additional safety measure, specific alert thresholds for individual physiological parameters have also been set. Study investigators will remotely review the individual subject's physiological parameters every 4 hours, and order diagnostic tests for COVID-19 infection through instant communication (electronic communication and/or phone). In addition, after the initial

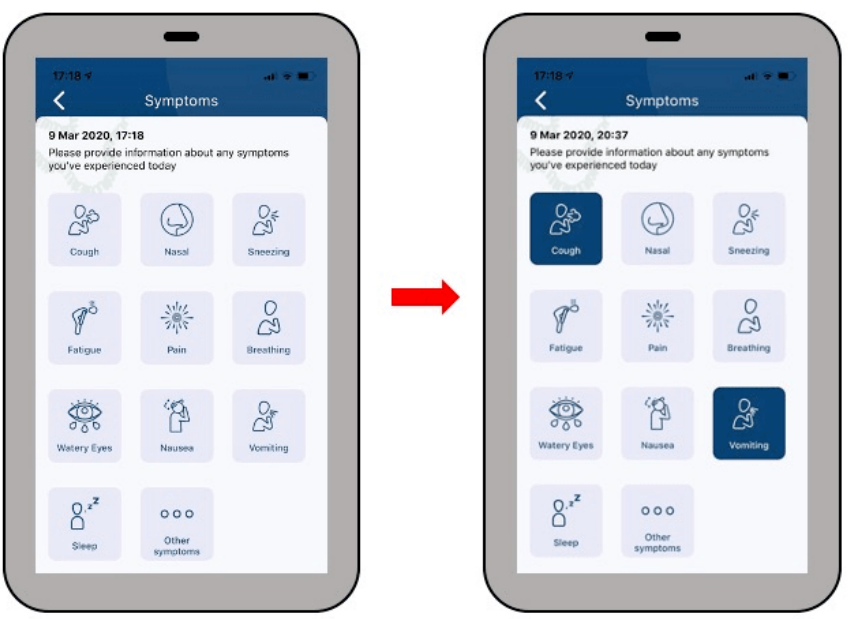

Figure 3 Smartphone application screen for symptom surveillance.

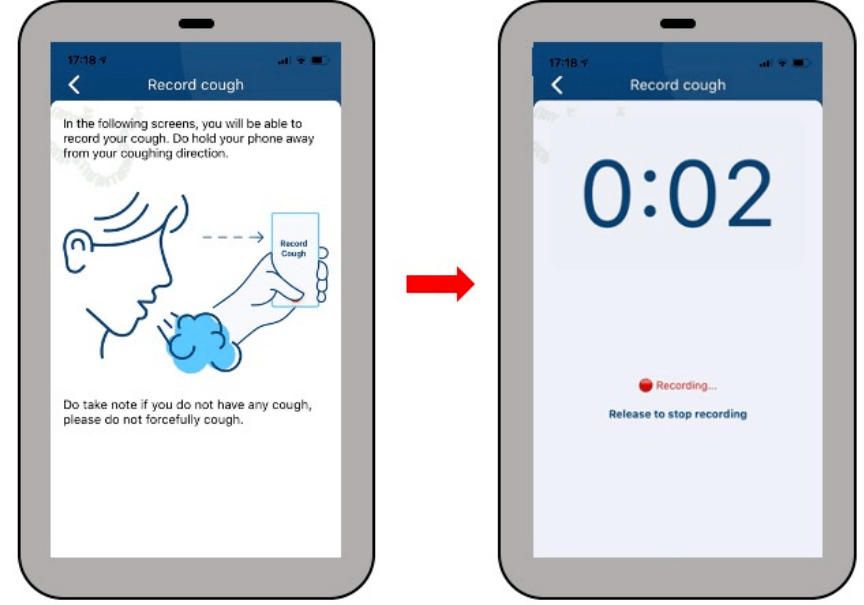

Figure 4 Smartphone application screen for cough sound recording.

run-in phase and having recruited an adequate number of subjects with subsequently confirmed COVID-19, the collected physiological data will be used to enhance the Biovitals prediction model for COVID-19 infection using machine learning technology.

\section{Outcomes}

The primary outcome measure is the time to detection of COVID-19 infection using nasopharyngeal sampling for COVID-19 reverse transcription polymerase chain reaction (RT-PCR).

The secondary outcomes include wearable device adherence, sensitivity, specificity, positive predictive value, negative predictive value and the area under the receiver operating characteristic curve of Biovitals Sentinel in identifying COVID-19 subjects, viral load of COVID-19 using nasopharyngeal sampling for RT-PCR at diagnosis, crossinfection rate within family cluster, length of hospital stay, length of intensive care unit stay, non-invasive and invasive ventilation use, National Early Warning Score 2, worsening of comorbidities and mortality.

\section{Sample size and statistical analysis}

The sample size for the randomisation phase will be determined based on the result from the phase I run-in period involving approximately 100 subjects. The sample size of the run-in period was determined by convenience sampling because there were no previous researchthemed studies. To explore and estimate the effect of longitudinally measured clinical and physiological data on the time-to-event of primary and secondary outcomes,

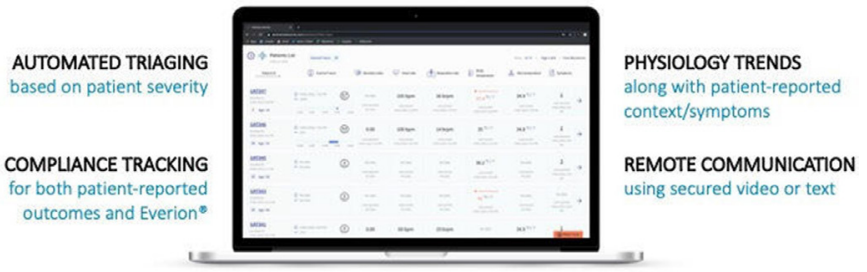

Figure 5 Web-based dashboard for clinicians. 
joint modelling of longitudinal and time-to-event data analysis, and other statistical and/or machine learningbased methods will be used. Data normality of continuous variables will be assessed using skewness statistics. Baseline characteristics of the two study groups will be compared using analysis of variance, $\chi^{2}$ or Fisher's exact tests, as appropriate. Analysis of the primary and secondary outcomes will conform to the intention-to-treat principle. Cox regression analysis will be performed to compare the time to diagnosis of COVID-19 infection between the study groups with adjustment for potential confounders. For other secondary outcomes, the generalised estimating equations model will be used to compare the differential changes in each of the outcomes across the 14-day time points between the two study arms, with adjustment for potential confounders.

\section{Randomisation}

Patients will be randomised either to the Intervention group or Control group using a computer-generated random number to derive allocation sequence prior to enrolment in the study. Study staff responsible for enrolment will be informed of the randomisation assignment after entering the information of the subjects to a designated computer system. If a subject is found to be ineligible for the study after randomisation, the original assignment will be reassigned to the next eligible subject. Subjects and clinicians will not be blinded to the randomisation assignment. Data staff responsible for data entry will be blinded from randomisation assignment.

\section{Data collection and management}

After enrolment, each subject will be assigned a unique identifier to be used in the database. Data will be entered by study staff and data accuracy will be verified by the principal investigator of the study. Data quality control measures include queries to identify missing data, outliers and discrepancies. The database will be password protected and encrypted. Only study staff will have access to the database. Subjects who withdraw from the study will have continuous monitoring stopped, usual care continued and final outcome collected for analysis.

\section{Data monitoring}

Due to the minimal risk nature of the study, there is no external data and safety monitoring board. The principal investigator and study staff will monitor data internally and meet weekly in person or by phone to ensure the study is proceeding as intended.

\section{Patient and public involvement}

We received inputs from quarantined individuals and healthcare providers which guided the design of the current study and the choice of research questions. No quarantined individuals or the public were directly involved in the design of the study and choice of outcome measures. No quarantined individuals or the public will be involved in recruitment or conduct of the study.
Results of the study will be disseminated to subjects, the public and the scientific community.

\section{ETHICS AND DISSEMINATION}

The investigation conforms to the principles outlined in the Declaration of Helsinki. The study protocol has been approved by the Institutional Review Board of The University of Hong Kong, and Hong Kong West Cluster, Hospital Authority, Hong Kong. Written informed consent will be obtained from all study participants by the study staff responsible for recruitment (online supplementary files 1 and 2). Important protocol modifications will be conveyed to investigators, the institutional review board, trial registries, regulators, journals and trial participants. After enrolment, each subject will be assigned a unique identifier to be used in the database. The personal identity of the subjects will not be used for any public purpose or for publication, nor will it be transmitted outside of the study team.

The data set used during the study will be available from the corresponding author upon reasonable request. Collaboration with other investigators interested in optimising quarantine strategies for COVID-19 will be welcomed. The results of the trial will be published in peer-reviewed journals and presented in conferences.

\section{DISCUSSION}

Emerging in December 2019, COVID-19 has spread at a rate far outstripping the capacity of many medical systems. ${ }^{14}$ Traditionally, public health measures including isolation and quarantine are the cornerstone to curb the spread of infectious diseases by interrupting personto-person transmission, and are particularly important when no specific therapeutics or vaccines are available. ${ }^{6}$ Indeed, early detection of infected individuals among those with viral exposure followed by isolation would effectively reduce overt viral shedding in the community; nonetheless, early detection can be challenging.

Symptom and fever surveillance among individuals with COVID-19 exposure is a commonly used method to detect infected individuals in the absence of medical testing. However, symptom declaration and intermittent body temperature measurement per se may not be sufficient to detect early disease progression. Indeed, up to $50 \%$ COVID-19 infected patients were so-called 'asymptomatic' and did not have fever until the full-blown disease. ${ }^{7}$ Conceivably, while fever is one of the cardinal symptoms specific for active infection, many other physiological alterations such as heart rate, respiratory rate, oxygen saturation, perspiration and so on may likewise indicate active infection. However, practically speaking, they are more difficult to accurately measure without medical training.

The intervention arm of the current study differs from the existing symptom and fever surveillance-based quarantine programme in several important aspects. By 
incorporating a wide range of continuously collected physiological parameters instead of solely relying on intermittent body temperature, it is possible to more accurately assess an individual's physiological changes for detecting early disease progression. ${ }^{9}$ At the same time, the use of contemporary mobile communication technology facilitates automatic collection and transfer of physiological data. These measures can enhance patient compliance with the monitoring system as well as greatly shorten the time between detection of an abnormality and subsequent intervention. In the past decades, wearable technology has been increasingly used for medical diagnosis, most notably in arrhythmia detection. ${ }^{15-21}$ Indeed, by continuously collecting multidimensional physiological parameters and analysing them with machine learning techniques, it is possible to acquire a much more in-depth understanding of different disease processes, which potentially allows us to improve clinical management. Nonetheless, such potentials have not been fully explored in real world disease management. In the short term, the results of the current study may provide insights into detecting disease progression of COVID-19 through physiological data monitoring and possible ways to reduce the spread of the disease. In the long term, the study may demonstrate an important example of how wearable technologies and machine learning techniques can be incorporated into existing medical services.

The clinical trial is limited by its single-centred characteristic and being exploratory in nature.

\section{Author affiliations}

${ }^{1}$ Division of Cardiology, Department of Medicine, University of Hong Kong, Hong Kong, Hong Kong

${ }^{2}$ Division of Infectious Diseases, Department of Medicine, University of Hong Kong, Hong Kong, Hong Kong

${ }^{3}$ Harmony Medical Inc, Hong Kong, Hong Kong

${ }^{4}$ Biofourmis, Singapore

${ }^{5}$ Research and Development, Biofourmis, Singapore

Acknowledgements The authors thank Biofourmis, Singapore, and Harmony for donating the Everion wearable devices. The authors also thank PCCW for supporting the mobile phone and cellular data.

Contributors CWS, IFNH, CKW, RCFT and KSR contributed to the conception and design of the study. DTYH, ART, MZ and MOYT contributed to the acquisition of data. Data analysis and interpretation will be conducted by CWS, IFNH, CKW, RCFT, KSR, GC, SCC and YML. CWS, IFNH and CKW wrote first draft of the protocol. ART, MZ and YML revised the protocol critically for important intellectual content. All authors have read and approved the final version of the manuscript to be published.

Funding The authors have not declared a specific grant for this research from any funding agency in the public, commercial or not-for-profit sectors.

Competing interests RCFT is employed by Harmony Medical, which donated the Everion wearable devices. KSR, GC and SCC are employed by Biofourmis, which donated the Everion wearable devices.

Patient consent for publication Not required.

Provenance and peer review Not commissioned; externally peer reviewed.

Open access This is an open access article distributed in accordance with the Creative Commons Attribution Non Commercial (CC BY-NC 4.0) license, which permits others to distribute, remix, adapt, build upon this work non-commercially, and license their derivative works on different terms, provided the original work is properly cited, appropriate credit is given, any changes made indicated, and the use is non-commercial. See: http://creativecommons.org/licenses/by-nc/4.0/.

\section{ORCID iDs}

Chun Ka Wong http://orcid.org/0000-0001-5205-9440

Mi Zhou http://orcid.org/0000-0002-0864-9512

\section{REFERENCES}

$1 \mathrm{Li} \mathrm{Q}$, Guan X, Wu P, et al. Early transmission dynamics in Wuhan, China, of novel coronavirus-infected pneumonia. N Engl J Med 2020;382:1199-207.

2 Wu JT, Leung K, Leung GM. Nowcasting and forecasting the potential domestic and international spread of the 2019-nCoV outbreak originating in Wuhan, China: a modelling study. Lancet 2020;395:689-97.

3 Guan WJ, ZY N, Hu Y, et al. Clinical characteristics of coronavirus disease 2019 in China. N Engl J Med 2020.

4 Chan JF-W, Yuan S, Kok K-H, et al. A familial cluster of pneumonia associated with the 2019 novel coronavirus indicating personto-person transmission: a study of a family cluster. Lancet 2020;395:514-23.

5 Dong E, Du H, Gardner L. An interactive web-based dashboard to track COVID-19 in real time. Lancet Infect Dis 2020;20:533-4.

6 Hellewell J, Abbott S, Gimma A, et al. Feasibility of controlling COVID-19 outbreaks by isolation of cases and contacts. Lancet Glob Health 2020;8:e488-96.

7 Kenji Mizumoto KK, Zarebski A, Chowell G. Estimating the asymptomatic proportion of 2019 novel coronavirus onboard the Princess cruises SHIP. medRxiv 2020.

8 van Vliet M, Donnelly JP, Potting CMJ, et al. Continuous non-invasive monitoring of the skin temperature of HSCT recipients. Support Care Cancer 2010;18:37-42.

9 Pevnick JM, Birkeland K, Zimmer R, et al. Wearable technology for cardiology: an update and framework for the future. Trends Cardiovasc Med 2018;28:144-50.

10 Chan A-W, Tetzlaff JM, Altman DG, et al. Spirit 2013 statement: defining standard protocol items for clinical trials. Ann Intern Med 2013;158:200-7.

11 Chan A-W, Tetzlaff JM, Gøtzsche PC, et al. Spirit 2013 explanation and elaboration: guidance for protocols of clinical trials. $B M J$ 2013;346:e7586.

12 Altman DG, Schulz KF, Moher D, et al. The revised consort statement for reporting randomized trials: explanation and elaboration. Ann Intern Med 2001;134:663-94.

13 Moher D, Schulz KF, Altman DG, et al. The CONSORT statement: revised recommendations for improving the quality of reports of parallel group randomized trials. BMC Med Res Methodol 2001;1:2.

14 Camps JA, Zuur C, Blokland JA, et al. A breathing lung phantom for $81 \mathrm{mKr}$ lung ventilation studies its use in dosimetry and quality control. Eur J Nucl Med 1988;14:529-32.

15 Guo $\mathrm{Y}$, Wang $\mathrm{H}$, Zhang $\mathrm{H}$, et al. Mobile photoplethysmographic technology to detect atrial fibrillation. J Am Coll Cardiol 2019;74:2365-75.

16 Perez MV, Mahaffey KW, Hedlin $\mathrm{H}$, et al. Large-scale assessment of a smartwatch to identify atrial fibrillation. $N$ Engl $J$ Med 2019;381:1909-17.

17 Poh M-Z, Loddenkemper T, Swenson NC, et al. Continuous monitoring of electrodermal activity during epileptic seizures using a wearable sensor. Conf Proc IEEE Eng Med Biol Soc 2010;2010:4415-8.

18 Poh M-Z, Loddenkemper T, Reinsberger C, et al. Convulsive seizure detection using a wrist-worn electrodermal activity and accelerometry biosensor. Epilepsia 2012;53:e93-7.

19 Chan P-H, Wong C-K, Pun L, et al. Diagnostic performance of an automatic blood pressure measurement device, Microlife WatchBP home a, for atrial fibrillation screening in a real-world primary care setting. BMJ Open 2017;7:e013685.

20 Chan P-H, Wong C-K, Pun L, et al. Head-to-head comparison of the AliveCor heart monitor and microlife WatchBP office AFIB for atrial fibrillation screening in a primary care setting. Circulation 2017;135:110-2.

21 Chan P-H, Wong C-K, Poh YC, Chan Pak-Hei, Wong Chun$\mathrm{Ka}$, et al. Diagnostic performance of a smartphone-based Photoplethysmographic application for atrial fibrillation screening in a primary care setting. J Am Heart Assoc 2016;5:e003428. 